\title{
Ciencia y Tecnología: una mirada desde la Ingeniería
}

Esp. Cuevas, Alicia; Esp. Amarilla, Alicia; Mgter. Corvalán, Rubén ( ${ }^{*}$ )

\section{Resumen}

Los avances producidos en los ámbitos de la ciencia y la tecnología que habían tenido su mayor auge en los albores del siglo pasado, hoy a la luz del nuevo contexto sanitario-epidemiológico global, han experimentado un despegue inusitado que involucra a gran parte de la población mundial. La Ingeniería como estimuladora de nuevas soluciones en un mundo por descubrir, ha sido el artífice para crear esta realidad. Miremos juntos como esta tríada: Ciencia, Tecnología e Ingeniería, se interrelacionan e imbrican hasta conformar el escenario de nuestro siglo XXI.

Summary: The advances produced in the fields of science and technology that had had their greatest boom at the dawn of the last century, today in light of the new global sanitary-epidemiological context, have experienced an unusual take-off that involves a large part of the world population . Engineering as a stimulator of new solutions in a world to be discovered, has been the architect to create this reality. Let's look together at how this triad: Science, Technology and Engineering, interrelate and overlap to form the scenario of our 21st century.

(*) Grupo GRATOS: Grupo Regional de Administración, Tecnología, Organización y Sociedad. Departamento de Ingenieria. Facultad de Ciencias Exactas y Naturales y Agrimensura. Av. Libertad 5470. Corrientes. Mail: alisucuevas@ yahoo.com.ar 
Palabras Clave: Métodos, Desarrollo, Innovación, Cibercultura, Globalización.

Key Words: Methods, Development, Innovation, Cyberculture, Globalization.

El ser humano desde su aparición en el mundo siempre hizo cosas técnicas que fueron la Ingeniería de su tiempo. De acuerdo a las actividades que realizaba, fue nominado: Homo Sapiens; el que pretende conocer la realidad y comprender los fenómenos de su tiempo y Homo Faber: el que construye e inventa herramientas rudimentarias para satisfacer las necesidades que le permitan sobrevivir en su medio. La tecnología pasa luego por el Ingenio medieval (especie de catapulta) donde se produjo un salto en lo que respecta a maquinaria bélica, y posterior a sucesivas evoluciones a lo largo de los años, hoy podemos apreciar ese salto en los robots que trabajan bajo el conjuro de la informática asociada a la máquina. Es por ello que la Ingeniería ha sido vista como la expresión creativa de la Ciencia y la Tecnología por medio del desarrollo de nuevas herramientas, maquinaria, estructuras y toda innovación que surja en las diferentes orientaciones y especialidades que de ella nacen.

\section{¿De qué hablamos cuando nos referimos a Ingeniería, Ciencia y Tecnología?}

Para ello se hace necesario conocer su etimología y algunas definiciones: la palabra Ingeniería proviene del latín Ingenium: facultad de una persona para inventar con prontitud o solucionar algo con facilidad; y del inglés Engine: ingenio, invento o máquina fabricada. Se denomina Ingeniería al conjunto de conocimientos y técnicas científicas aplicadas a la creación e implementación de estructuras físicas y teóricas para la resolución de problemas de la sociedad

Según el CONFEDI (Consejo Federal de Decanos de las Facultades de Ingeniería) "la Ingeniería es la profesión en que el conocimiento de las ciencias matemáticas y naturales adquiridas mediante el estudio, la experiencia y la práctica: se emplea con buen juicio a fin de desarrollar modos en que se puedan utilizar, de manera óptima los materiales y las fuerzas de la naturaleza en beneficio de la hu- 
manidad; en el contexto de restricciones: éticas, físicas, económicas, ambientales, humanas, políticas, legales y culturales" (tomado de ABET: Acreditatión Board of Engineering and Technology. USA 2001)

A su vez la práctica de la Ingeniería comprende el estudio de factibilidad técnico económico, la investigación, desarrollo e innovación, diseño, proyecto, modelación, construcción; optimización, evaluación, gerenciamiento, dirección y operación de todo tipo de componentes, equipos, máquinas, instalaciones, edificios, sistemas y procesos. Lo que distingue al Ingeniero en su práctica además del conocimiento y la experiencia, es su imaginación y creatividad, su capacidad de proponer soluciones alternativas e innovadoras puestas al servicio de la sociedad.

La Ingeniería pretende entender lo mejor posible la realidad que debe enfrentar, para prestar un servicio o crear un producto con suficiente seguridad, optimizando la calidad del mismo con los recursos disponibles y todo ello al menor costo.

Por su parte se denomina Ciencia al conjunto de conocimientos verdaderos que ha evolucionado a través del tiempo, siendo su objetivo principal la búsqueda del saber. La palabra Ciencia proviene del latín Scientia: que significa conocimiento, el cual se presenta de manera ordenada y sistematizado.

Es decir la Ciencia es una rama del saber humano, constituida por un conjunto de actividades que de manera metódica y organizada, busca saber el porqué de las cosas para satisfacer una curiosidad. Esto genera como resultado conocimientos racionales, objetivables y verificables, mediante la observación, la formulación de hipótesis con su posterior verificación mediante la experimentación; determinando la relación causa - efecto, y los principios y leyes que gobiernan la naturaleza.

En tanto que la palabra Tecnología proviene del griego, Téchne: arte u oficio y de Logía: tratado o estudio; y constituye el conjunto de conocimientos técnicos y procesos científicos, que permiten conocer cómo son las cosas, para diseñar y construir objetos tecnológicos que satisfagan las necesidades humanas o mejoren la calidad de vida. 
De esto se desprende que la Tecnología es una actividad orientada a producir máquinas, aparatos y sistemas; que resultan de vincular el saber y el hacer; por medio de procedimientos que combinan creatividad, arte y técnica, que resultan en productos y prestaciones de utilidad económica, política y social. Son los Ingenieros quienes generan y operan las tecnologías.

\section{Diferencias entre la Ciencia y la Tecnologia}

\begin{tabular}{l|l|}
\multicolumn{1}{c|}{ Ciencia } & \multicolumn{1}{c|}{ Tecnología } \\
\hline PROPÓSITQ: Explicación & PROPÓSITO: Producción \\
INTERÉS: Lo natural & INTERÉS: Lo artificial \\
PROCESO: Analítico & PROCESO: Sintético \\
PROCEDIMIENTO: Simplificación del fenómeno & PROCEDIMIENTO: Complejidad de la necesidad \\
RESULTADO: Conocimientos qeneralizables & RESULTADQ: Objeto particular \\
\hline
\end{tabular}

En tanto que se denomina Técnica al conjunto de procedimientos o recursos que se usan con buen arte, en una actividad determinada, para la cual se requiere cierta habilidad que se adquiere con la práctica. La palabra proviene del latín: Technicus y esta su vez del griego: Txun que significa arte. Se puede deducir entonces como el arte de transformar la naturaleza elemental que rodea al ser humano en función de sus necesidades, con el fin de crear un mundo artificial, según sus expectativas; con sus beneficios y también con algunas dificultades asociadas; las cuales influyen sobre su vida personal y social.

Si consideramos las interacciones que se producen en la tríada: Ingeniería, Ciencia y Tecnología; podemos establecer cómo serían las relaciones que las interconec$\tan$.

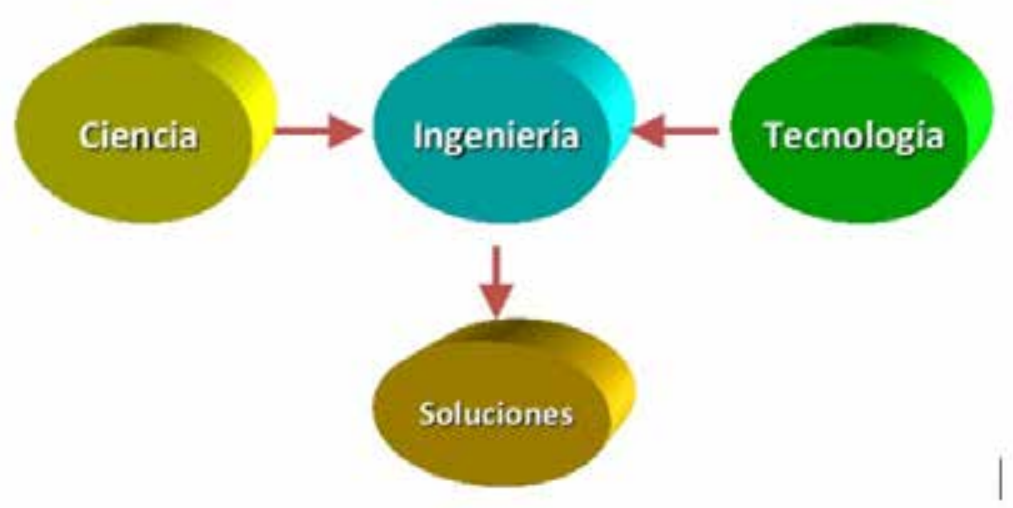

Figura 1 : Interacciones de la triada 
La Ingeniería pues resulta de combinar la dosis adecuada de Ciencia, Arte y Tecnología; Ciencia enriquecida por el Arte y Tecnología humanizada por el Arte. Pero a su vez la Ciencia constituye una herramienta que permite a la Ingeniería crear y operar Tecnología. De aquí que la Ciencia y la Tecnología son componentes centrales de cambio y desarrollo para las sociedades y de ahí el papel fundamental de la Ingeniería.

A pesar de las relaciones que tienen lugar entre la Ciencia y la Ingeniería; también pueden observarse diferencias en cuanto a sus métodos, objetivos y procedimientos.

Se puede decir que el método científico es un proceso destinado a observar fenómenos, tratar de establecer relaciones entre los hechos, reproducirlos y enunciar leyes que los expliquen y con estos conocimientos generar aplicaciones que sean útiles al hombre.

Por su parte el método ingenieril es una actividad de toma de decisiones ante las limitaciones o carencias con el fin crear productos materiales o procesos que satisfagan esa necesidad.

Métodos de la CIENCIA

Ansias de Conocimiento<smiles></smiles>

Investigación Cientifica<smiles></smiles>

Conocimientos Científicos
Métodos de la INGENIERIA

Satisfacción de necesidades

$\downarrow$

Desarrollo, diseño, ejecución y operación

$\downarrow$

Bienes, servicios, métodos o procesos

De acuerdo al cuadro anterior del Profesor argentino Aquiles Gay, se puede deducir que la Ciencia busca conocer y para ello estudia lo que ya existe en la naturaleza; en tanto que la Ingeniería busca realizarse por medio de la creación de lo que no existe. 


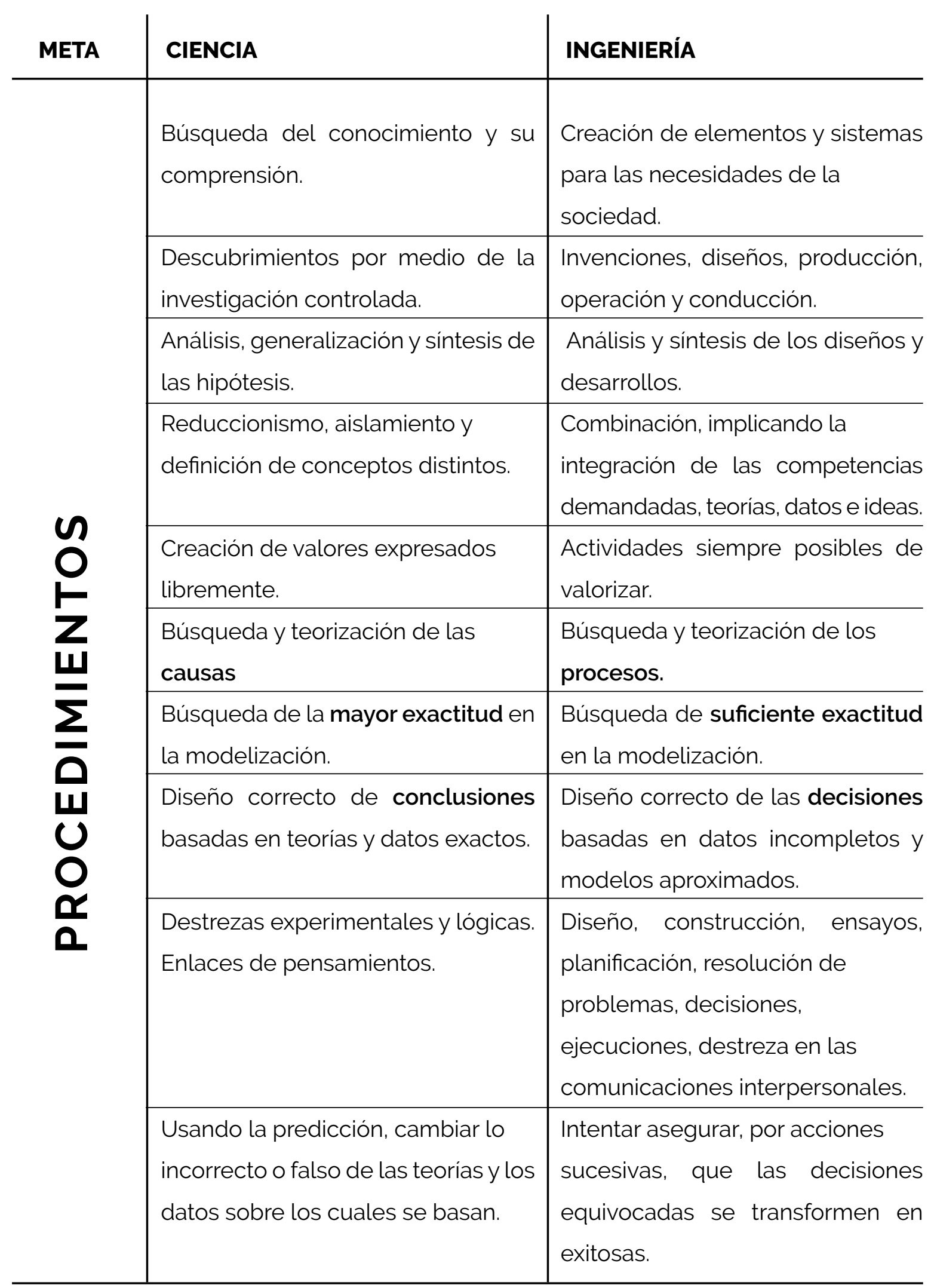




\section{Desarrollo del Método Científico y sus diferencias con el Modelo Ingenieril}

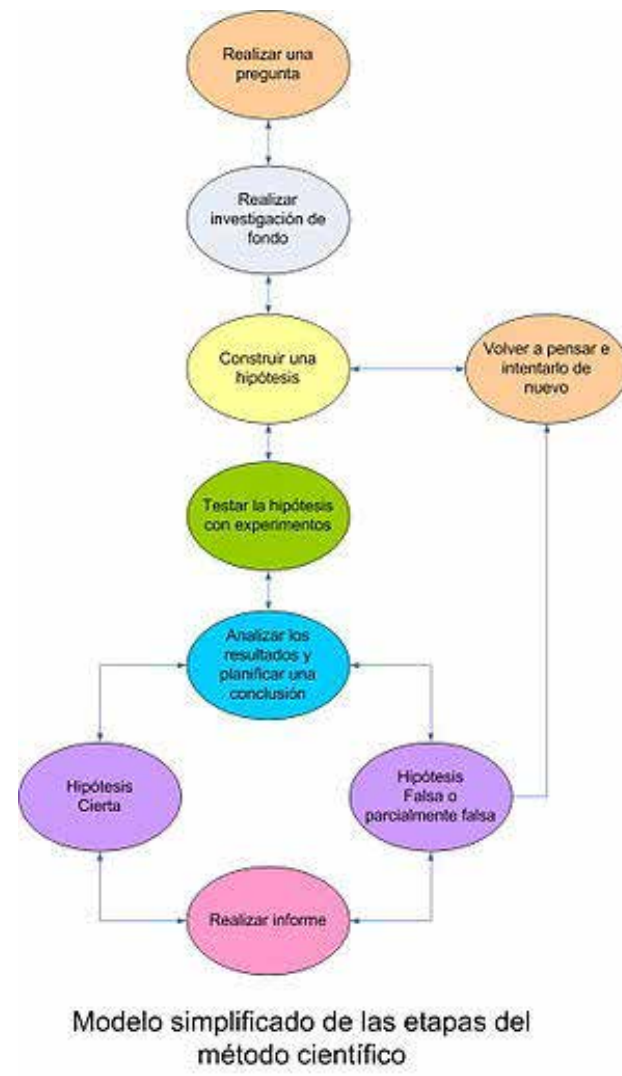

Figura 2 : Método Científico.

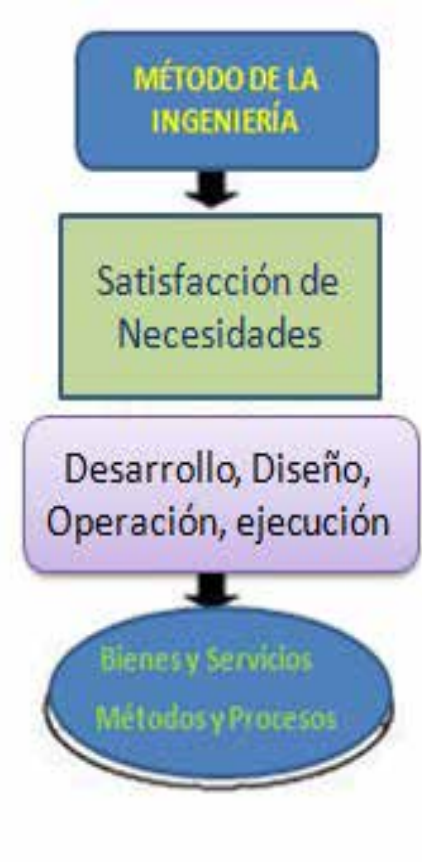

FIgura 3 : Método de la Ingeniería

El Método Ingenieril busca satisfacer una necesidad, ante una carencia o falta de un bien o servicio considerado esencial para lograr el bienestar de manera individual o colectiva; o bien por la ausencia del requerimiento necesario para lograr un nivel de vida o propósito; todo lo cual es percibido como un problema cuya solución demandará la aplicación de éste método común a toda las Ingenierías.

\section{Investigación, desarrollo e innovación}

$\mathbf{I}+\mathbf{D}+\mathbf{i}$ : es un concepto que proviene de los estudios de ciencia, economía y tecnología.

Del mundo de la Ciencia surge la Investigación, como una indagación original y planificada cuyo objetivo es descubrir nuevos conocimientos y lograr una 
mayor comprensión en el ámbito científico y tecnológico; se puede decir que es "invertir dinero para obtener conocimiento"

El Desarrollo es un término proveniente del mundo de la Economía, tomado como sinónimo de evolución y se refiere al proceso de cambio y crecimiento relacionado con una situación, individuo u objeto determinado.

A su vez la innovación procede de la Tecnología, como una actividad cuyo resultado es el avance tecnológico por medio de la creación o mejoría sustantiva de productos o procesos; es "invertir conocimiento para obtener dinero" Cuando alguien innova aplica nuevas ideas, conceptos, productos, servicios y prácticas a una determinada cuestión, actividad o negocio, con la intención de incrementar la productividad

Desarrollo Tecnológico: uso sistemático del conocimiento y la investigación dirigidos hacia la producción de materiales, dispositivos, sistemas o métodos incluyendo el diseño, desarrollo, mejora de prototipos, procesos, productos, servicios o modelos organizativos con el fin de alcanzar mejoras en términos de salud, producción industrial, telecomunicaciones, transporte, comercio, educación, industria militar y cualquier actividad. El desarrollo de alta tecnología ha permitido conquistar las barreras de comunicación, acortando lejanías geográficas, y en consecuencia el ritmo de vida ha aumentado.

Vinculación Tecnológica: es la relación que se establece entre quien transfiere y quien recibe conocimiento. Es el intercambio de conocimientos científico-tecnológicos generados en el ámbito académico universitario y que son transferidas o vertidas hacia el entramado social y productivo, de manera que sirvan al desarrollo, competitividad y crecimiento de las empresas y organizaciones del medio.

Cuando se habla de vinculación tecnológica, ésta puede ser interpretada por varios modelos de representación: uno de ellos es el Triángulo desarrollado por Jorge A. Sábato: conformado por 3 elementos: Estado, Universidad (I+D) y Estructura Productiva. El Triángulo de Sábato es un modelo de política científico-tecnológica, donde son necesarios: el Estado (como diseñador y ejecutor de la política), la infraestructura científico-tecnológica (como sector de oferta de tecnología) y el sector productivo 


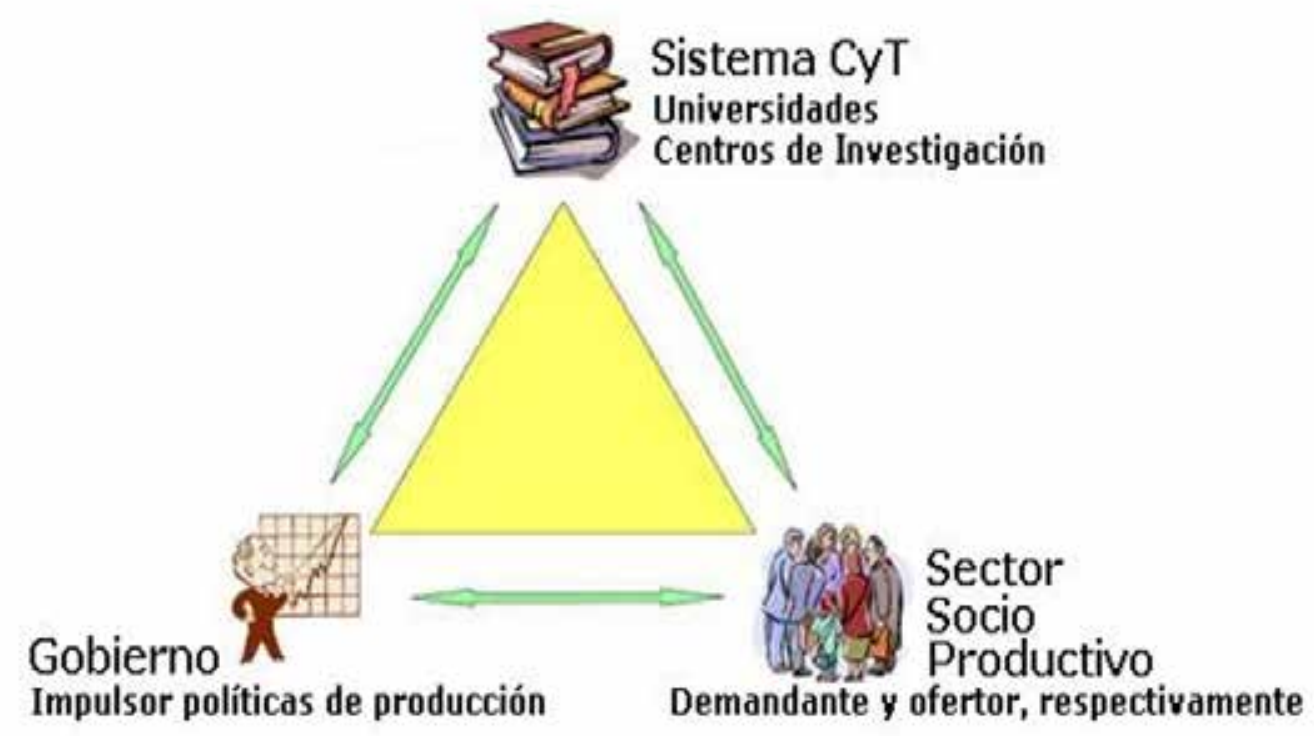

Figura 4: Triangulo de Sàbato

(como demandante de tecnología), todos los cuales están relacionados fuertemente de manera permanente.

Nodo del Conocimiento: es una segunda forma de modelización, donde se considera que la transferencia tiene lugar en los nodos o nudos de conocimiento, el cual es atravesado en diferentes direcciones por flechas que representan la generación, la transferencia propiamente dicha y la aplicación de la misma.

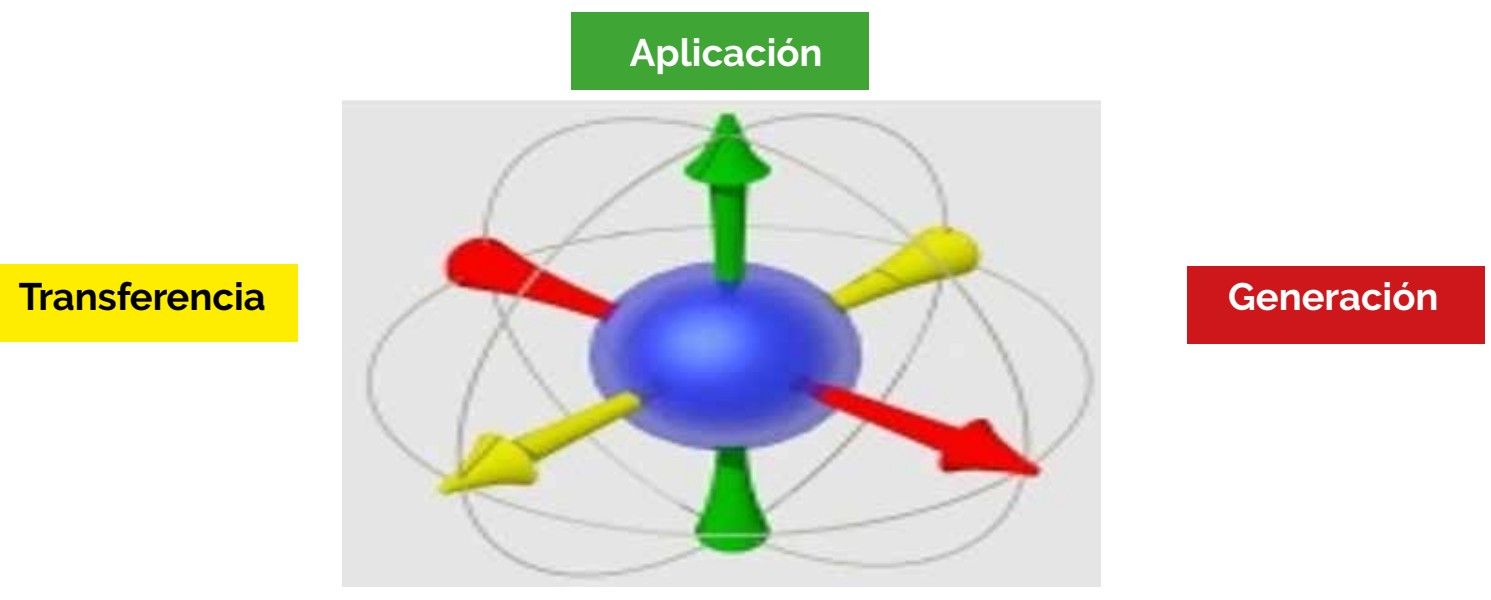

Figura 5: Nodo del Conocimiento. 
Triple Hélice o Código Genético: a su vez este modelo determina que la vinculación que se produce entre los diferentes sectores que interactúan: Universidad, Estado y Producción, toma una disposición con múltiples entrecruzamientos y puntos de contacto a manera del código de ADN de triple cadena.

\section{Universidad}

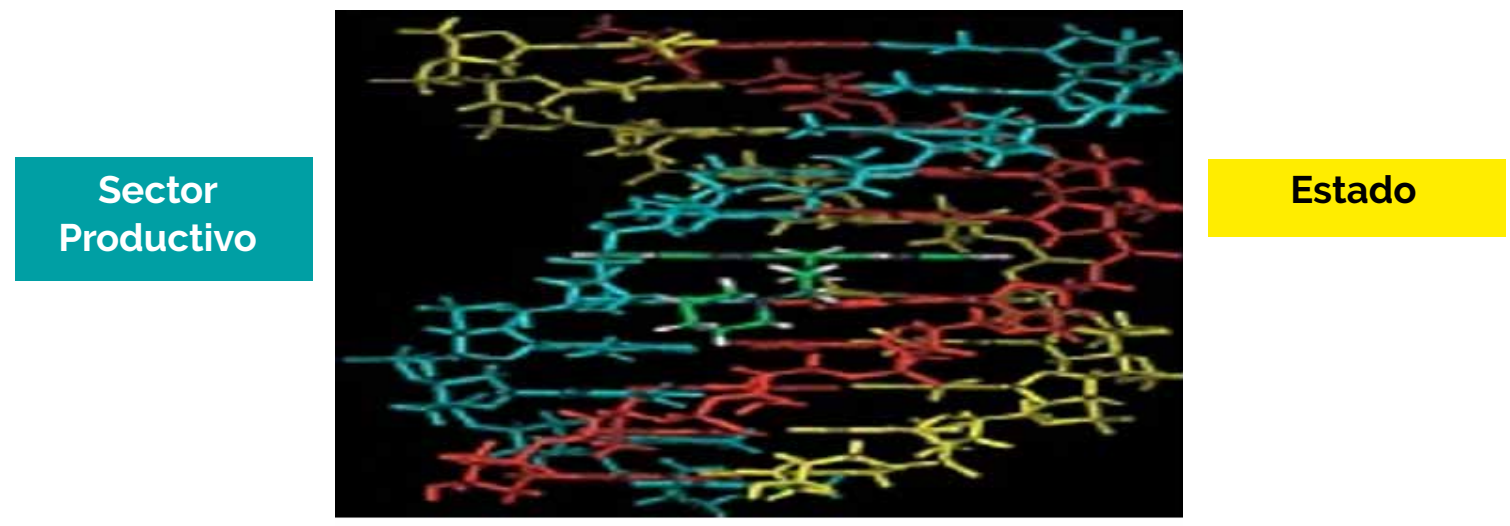

Figura 6: Triple Hélice.

\section{I+D+i y Transferencia Tecnológica local}

En el ámbito de la FaCENA (Facultad de Ciencias Exactas, Naturales y Agrimensura) de Corrientes funcionan los siguientes Grupos de Investigación en Ingeniería

- GER: Grupo de Energías Renovables

- GRATOS: Grupo Regional de Administración, Tecnología, Organización y Sociedad

- GTMyGRH: Grupo de Telemedicina y Gestión de Recursos Humanos

- GRIER: Grupo de Ingeniería en Rehabilitación

- GIATE: Grupo de Investigación Aplicada y Transferencia de Tecnología

- GIRNI: Grupo de Investigación en Medios No Ionizantes

- GDT: Grupo de Desarrollo Tecnológico

- GIB: Grupo de Ingeniería Biomédica

\section{¿Cómo llegamos a nuestros días?}

A través de la Historia el hombre ha atisbado el futuro, por medio de su pensamiento científico, creando con sus manos bienes y servicios, al resolver problemas relacionados con la satisfacción de una necesidad socio-cultural específica, lo que permitió a la humanidad dar saltos progresivos en su desarrollo, que a manera de olas generaron sucesivas revoluciones de progreso. 
Hasta el momento se han podido identificar cuatro revoluciones industriales las cuales posibilitaron años de avance tecnológico en un corto período de tiempo. Estos fueron los acontecimientos históricos que dan cuenta de ello:

La $1^{\circ}$ Revolución Industrial comprende entre 1760 y 1830; la cual tuvo lugar en Inglaterra con la invención de la máquina de vapor, que posibilitó pasar de la producción manual a la mecanización, lo que permitió aumentar la producción en la industria de los textiles y con ello favorecer la exportación hacia América y otros destinos de comercio; asimismo también se logró el movimiento de grandes máquinas para el transporte de personas: nacieron así los coches, locomotoras y barcos a vapor. Un pequeño grupo de ingenieros en Londres, formó la Sociedad de Ingenieros; y John Smeaton director del grupo, fue el primer Ingeniero "Civil" para señalar que su incumbencia no era militar, lo que inició una nueva especialización.

La Segunda Revolución Industrial se produjo a partir de 1850 y los albores del 1900, con el desafío de usar la electricidad como nueva fuente de energía, para la manufacturación en masa, sin que su manipulación implique un peligro para la vida del hombre.

Entre los inventores de esta etapa se destaca el científico italiano Alessandro Volta quién fabricó la primera pila eléctrica. Thomas Alva Edisson inventor de la bombilla eléctrica que al iluminar la sociedad de su tiempo, logró su máxima contribución al bienestar de la humanidad. El ingeniero eléctrico Nikola Tesla crea y patenta el sistema de corriente alterna, con la premisa de beneficiar con energía gratuita a la gente de su época. Las comunicaciones se agilizan por medio del telégrafo eléctrico inventado por Samuel Morse. Y en 1913 Henry Ford crea los clásicos automóviles: Ford T y Ford A; los cuales son producidos por medio de la línea automática de montaje; fabricando 10 millones de unidades, todo un logro de su tiempo.

La Tercera Revolución Industrial tiene lugar con el desarrollo de la electrónica a mediados del siglo XX, lo que supone el uso de componentes para controlar la electricidad. El desafío es procesar y mover conocimientos: lo que da origen a la Informática y las Telecomunicaciones. Lee De Forest inventó el tríodo, válvula electrónica que amplifica todo tipo de señales de audio, radio y tv, siendo considerado 
por ello el "Padre de la electrónica". Michael Faraday crea el primer motor eléctrico, que convierte la energía eléctrica en energía mecánica; siendo utilizado tanto en los procesos industriales, como en los electrodomésticos. Jack Kilby inventa el primer circuito integrado o microchip, de uso en lo equipos electrónicos. Las megacomputadoras dan paso a las minicomputadoras, y las comunicaciones toman vuelo propio por el desarrollo de Internet, dando lugar a una nueva cultura y mundo global.

La exploración espacial designa los esfuerzos del ser humano en estudiar el espacio y sus astros desde el punto de vista científico y de su explotación económica. Una nave espacial es una estructura de ingeniería aeronáutica. Los sistemas de propulsión son resultado de ingeniería mecánica. Los soportes móviles que transportan las astronaves hasta el lugar del lanzamiento son obras de ingeniería civil y electromecánica. Todos los sistemas de seguimiento y control automático de vuelo son obras de la ingeniería electrónica. Los materiales de la nave son resultado de la ingeniería metalúrgica. Los combustibles de propulsión son producto de la ingeniería química. Los sistemas de comunicaciones son obra de la ingeniería electrónica, y la informática está en todo.

Muestra de ello fue la participación de dos Ingenieros argentinos en la misión Apolo 11, que condujo al primer hombre en dar un paso en la luna y un gran paso para la humanidad, el 20 de julio de 1969. Uno es el Ing. César Sciammarella, que diseñó el tanque de combustible líquido del Saturno V, de reducidas dimensiones para lograr un peso mínimo crítico. El motor tenía programado dos encendidos, el
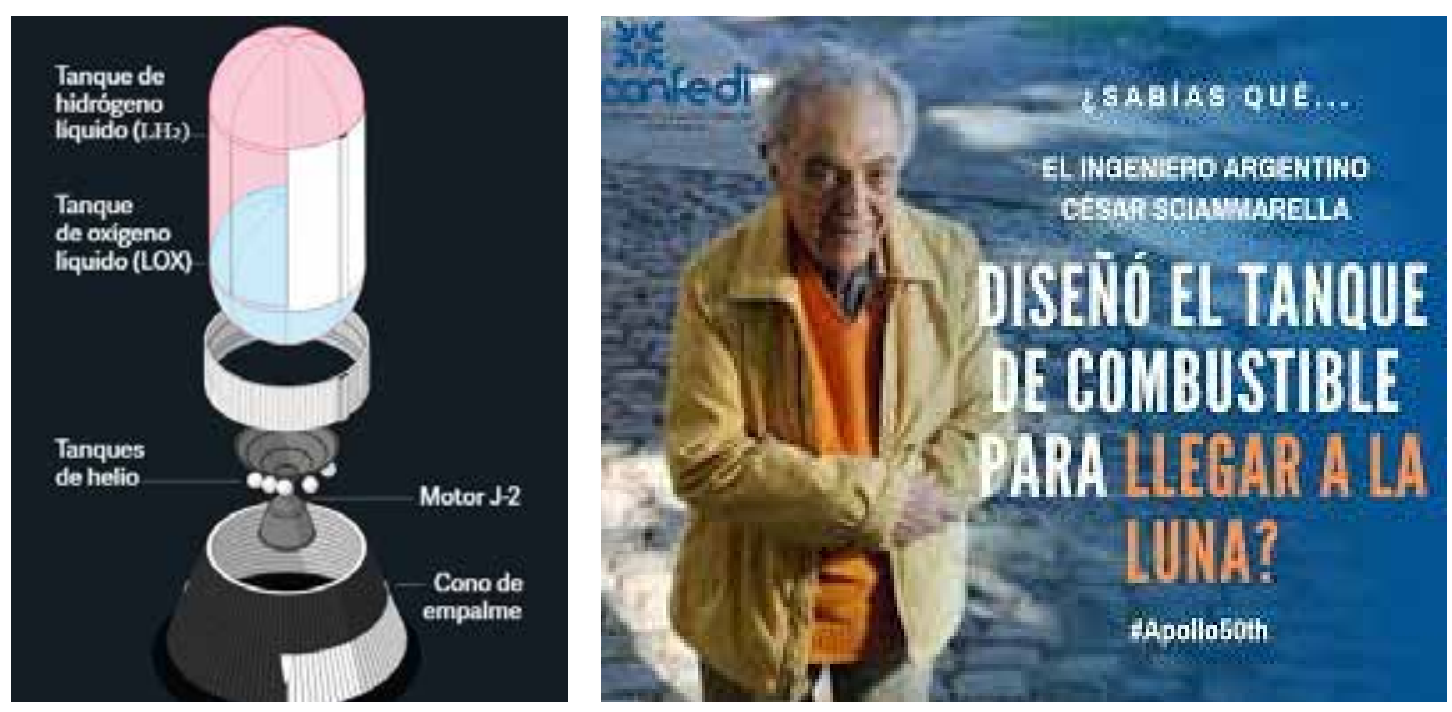


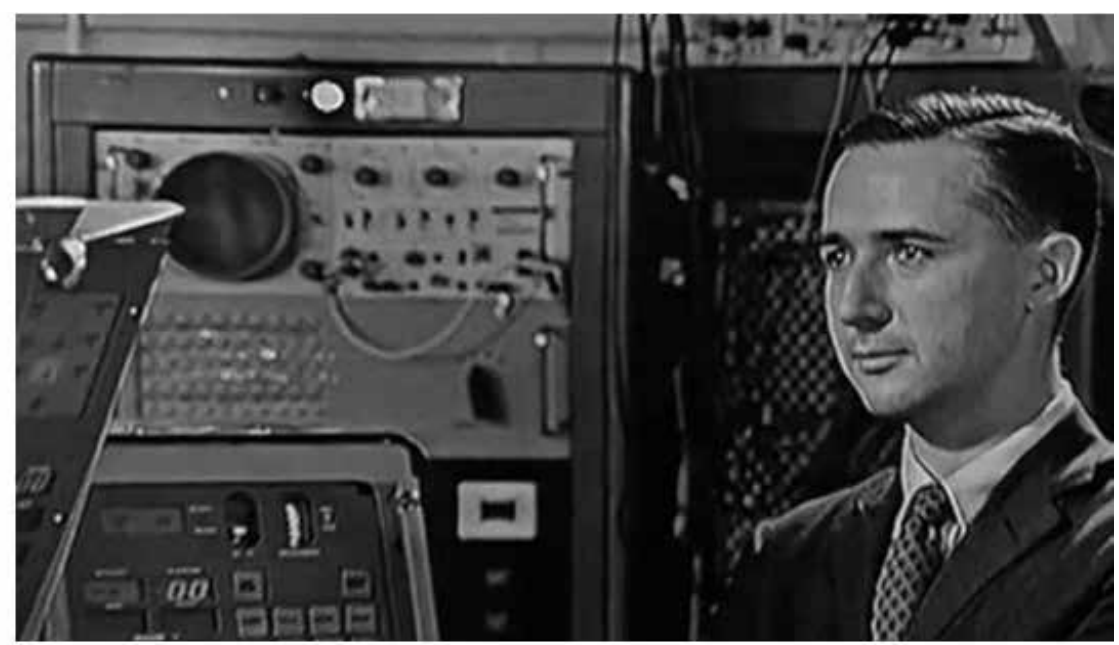

ALONSO. Ramón Alonso diseño la arquitectura de la computadora de la nave Apolo. (MIT)

primero para entrar en la órbita terrestre con una duración de $165 \mathrm{~s}$. y el segundo con una duración de 335 s. para realizar la inyección translunar de los módulos de mando y de servicio.

El otro argentino que participó del programa Apolo 11 fue el Ing. Ramón Alonso, que diseñó el hardware de la computadora de la nave, y de los módulos de comando y de alunizaje, lo que permitió el viaje y posterior alunizaje. Su trabajo se basó en circuitos integrados y memoria de soga que usaba imanes unidos con cables para codificar los datos, lo cual resistiría las altas temperaturas y radiaciones del espacio.

También creó la interfaz de la computadora, el teclado, el visor que usaron los astronautas y un sistema denominado DSKY (Display Keyboard) (comandos numéricos como verbos y sustantivos) en un teclado tipo calculadora.

\section{El despertar del mundo a una nueva cultura, al alcance de un byte.}

El desarrollo y expansión de los procesos tecnológicos e informáticos digitales, como medios de comunicación y de trabajo; originó una cultura cibernética, incorporada a una nueva sociedad.

Se denomina Cultura al conjunto de conocimientos, ideas, tradiciones y costumbres que caracterizan a un pueblo, a una clase social, o a una época. Proviene del latín Cultus, que significa cultivado en alusión a ilustrado o entendido sobre alguna 
cosa o tema. A medida que la cultura fue evolucionando, surgieron en el hombre nuevas necesidades a satisfacer que ya no fueran indispensables para su supervivencia, pero cuya satisfacción propiciaba una sensación de bienestar.

En tanto la voz de origen griego Ciber: deriva de kybernaein, que significa "manejar el timón, gobernar, pilotear, en referencia al espacio virtual creado por medios informáticos".

Por lo tanto la Cibercultura: es una nueva relación que se produce entre el hombre y el conocimiento.

Es un aporte más a las transformaciones sustanciales de la forma de vivir, aprender, producir y comerciar de casi toda la humanidad. Es una cultura asociada a la utilización de las nuevas tecnologías de las redes informáticas, empleadas para la comunicación, el entretenimiento y el mercado electrónico.

La Ingeniería también se identifica con esto y podemos decir que hoy estamos transitando la edad digital o de la tecno Ingeniería. Con la Cibercultura una persona con su PC o celular está conectada a una gran red mundial de comunicaciones por satélite y fibra óptica, como MySpace, Fotolog o Facebook.

\section{Características de la Cibercultura:}

1-La Interactividad: es la relación entre la persona y el entorno digital definido por el hardware que los conecta a los dos.

2-La Hipertextualidad: es el acceso interactivo a cualquier cosa que se da desde cualquier parte.

3-La Conectividad: es un estado humano comprendido por un mínimo de dos personas en contacto entre sí de modo virtual (existe de forma aparente y no es tangible).

A su vez esta tecnocultura tiene lugar y se desarrolla en el Ciberespacio, definido como el soporte para las tecnologías intelectuales o espacio virtual creado con medios cibernéticos. 
El término ciberespacio fue creado en 1982 por William Gibson en su novela "Neuromante" para referirse al lugar o espacio entre los teléfonos donde se produce la conversación telefónica. Vivimos en el mundo de las telecomunicaciones, la información, las redes de ordenadores, Internet; y este ciberespacio o universo eléctrico, tiene más entidad de la que tenía una simple conversación telefónica.

Dentro de las características del ciberespacio están: la identidad, la flexibilidad y el anonimato. La falta de interacción física cara a cara, causa un impacto en cómo la gente muestra su identidad; pudiendo presentarse bajo una apariencia imaginaria o falsa, cercanas al delito como el grooming. Las distancias geográficas no limitan quién puede comunicarse con quién, creando un espacio temporal donde el tiempo interactivo se extiende.

Suele asociarse el ciberespacio con la Internet, y a los usuarios de la red se los denomina Cibernautas, por lo tanto aquel que navega en esta realidad virtual mediante el uso de una computadora u ordenador, o bien desde otro dispositivo electrónico, como una tablet o un teléfono móvil celular, es un cibernauta. Para acceder a este espacio virtual utilizan un software conocido como navegador que les permite visitar los diferentes sitios web y utilizar servicios online; algunos de los navegadores más conocidos son el Explorer, Mozilla, Firefox y Chrome.

También se denomina Cibernauta a toda persona que utiliza los servicios informáticos del ciberespacio, que tienen actividad en las redes sociales, dejan comentarios en diferentes sitios web o participan de foros virtuales. El día 23 de agosto es cuando se celebra en todo el mundo el Día del Cibernauta, se eligió esa fecha para "recordar" el aniversario de la WWW (World Wide Web) o red mundial informática (1990).

El cibernauta busca el conocimiento donde está, para cada tema puede seleccionar el mejor profesor o la mejor bibliografía, con gran economía de tiempo, dinero y una eficiencia relevante. Estos procedimientos implican actuar en forma horizontal dentro de una gigantesca red interconectada, y a su vez al avanzar en el aprendizaje suministrar nuevos conocimientos a la red; este procedimiento estimula la inteligencia colectiva 
Esta era de la tecnología ha creado un hombre nuevo, conocido como nativo digital, término acuñado por el autor estadounidense Marc Prensky en 2001, por ser aquel que nace y se desarrolla dentro de un contexto que lo familiariza con la cultura de las nuevas tecnologías. Se consideran nativos digitales las personas nacidas desde el 1995 a 2015, siendo también conocida como la Generación Z.

Los nativos digitales enfocan su trabajo, el aprendizaje y los juegos de nuevas maneras: navegan con fluidez; tienen habilidad en el uso del mouse o ratón; utilizan reproductores de audio y video digitales a diario; toman fotos digitales que manipulan, editan y envían; y usan además sus ordenadores para crear videos y presentaciones.

Estos a su vez se distinguen de los immigrantes digitales, que son aquellas personas entre 35 y 55 años, nacidos en la era predigital antes de los años 90, que han tenido que aprender a usar los medios digitales proviniendo de un mundo analógico de información.

La humanidad, en la búsqueda del saber, sigue indagando nuevos caminos que lo ilustren cada vez más en su continuo progreso; incorporando esta nueva forma de pensar, aprender, trabajar, transmitir y producir conocimientos. Se dispone de nuevas herramientas ciberespaciales: los correos electrónicos que permiten una comunicación persona-persona, mucho más rápida.

La ciberseguridad o tecnología de la información fue creada con el propósito de brindar seguridad informática, se enfoca en la protección de la infraestructura computacional y todo lo relacionado con esta: la información contenida en una computadora o circulante a través de las redes de computadoras; comprende el software (bases de datos, metadatos, archivos) y el hardware, redes de computadoras y todo lo que la organización valore y signifique un riesgo si esa información llega a manos de otras personas.

Los bibliotecarios que usan los recursos del ciberespacio, dan lugar a un nuevo profesional: el Cibertecario o Cybarian (de asociar Cyberespace y Librarian), éstos términos fueron acuñados por Michel Bauwens. 
A su vez estas innovaciones en el campo de las telecomunicaciones y de la informática, por medio de internet, tuvo un rol decisivo en la construcción de un mundo globalizado.

La Globalización es proceso histórico de integración mundial en los ámbitos político, económico, social, cultural y tecnológico; como resultado de los principales avances tecnológicos, la consolidación del capitalismo y la necesidad de expansión del flujo comercial mundial en un lugar cada vez más interconectado; el mundo convertido en una aldea global.

La ruptura de las fronteras en términos económicos y de comunicación, generó una expansión capitalista en la que fue posible llevar a cabo transacciones financieras y expandir los negocios, hasta entonces limitados hacia otros mercados distantes.

El impacto de la Globalización en el aspecto económico: mercado laboral, comercio internacional; y en el aspecto político: instauración de sistemas democráticos, respeto de los derechos humanos; como así el acceso a la educación y la tecnología entre otros; varían en función del desarrollo de cada Nación, lo cual supondría en un futuro utópico el bienestar económico, la cohesión social y la libertad política.

Dentro de la Globalización tanto la Cibercultura como la Ingeniería, deben estar necesariamente asociadas a una conducta ética; de no ser así la humanidad corre el riesgo de caer en manos de minorías carentes de principios, creadoras de nuevas formas de esclavitud.

\begin{tabular}{|l|l|}
\hline \multicolumn{2}{|c|}{ GLOBALIZACIÓN } \\
\hline Ventajas & Desventajas \\
\hline Importación de bienes y productos & La riqueza se concentra en paises desarrollados \\
Aumento de inversiones extranjeras & Aumento de personas en la pobreza extrema \\
Potencia el comercio internacional & Causa aumento del desempleo \\
Propicia procesos de intercambio cultural & Pérdida de las identidades culturales tradicionales \\
Favorece el desarrollo tecnológico & Cultura global impuesta las grandes potencias \\
\hline
\end{tabular}


La Cuarta Revolución Industrial inicia en el año 2011, con la automatización total de la manufactura gracias a la organización de los procesos y medios de producción, por medio de sistemas ciberfisicos para llevar la producción a una total independencia de la mano de obra humana, mejorando el desarrollo tecnológico e industrial. Estos sistemas ciberfísicos, combinan maquinaria física y tangible con procesos digitales (internet o nube) y son capaces de tomar decisiones y de cooperar (entre ellos y con los humanos) dando lugar a una "fábrica inteligente". Las redes sociales permiten una nueva forma relacionamiento por medio de la virtualidad y las comunicaciones alcanzan su máxima expresión.

\section{Desarrollos de la Ingeniería:}

Inteligencia Artificial: es un programa de computación diseñado para auto-aprendizaje.

Domótica: es la tecnología aplicada al control y la automatización inteligente de la vivienda; lo cual permite una gestión eficiente del uso de la energía, aporta seguridad y confort, además de comunicación entre el usuario y el sistema.

Robótica: diseño y construcción de robots y aparatos que realizan trabajos; pueden ser de tipo industrial: brazo robótico o humanoide: e-Boy: niño robot. El escritor de ciencia ficción Isaac Asimov, crea las tres leyes de la robótica: 1-Un robot no hará daño a un ser humano, o por inacción, permitirá que un ser humano sufra daño. 2-Un robot debe cumplir las órdenes de los seres humanos, a excepción que entre en conflicto con la $1^{\circ}$ ley. 3 -Un robot debe proteger su propia existencia, en la medida que no entre en conflicto con las leyes previas.

Cibernética: es un mecanismo de control que regula los ingresos y egresos del sistema: input y output.

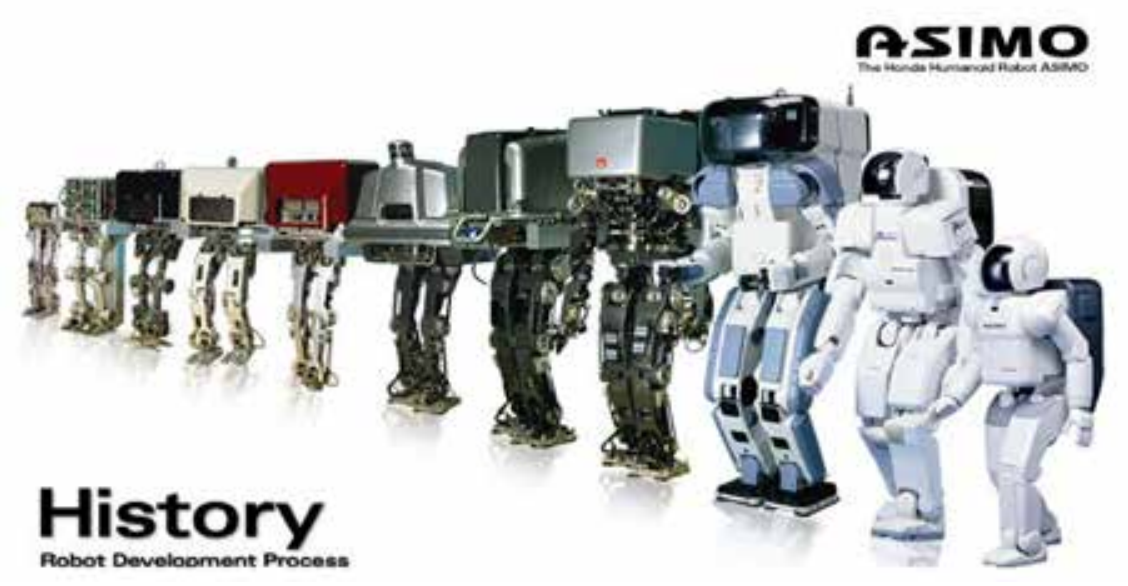


Biotecnología: comprende diferentes productos que derivan del uso de organismos vivos o sus partes, la química y la nanotecnología, entre otros.

Biomateriales: sustancias no farmacológicas en contacto con estructuras vivas, y dispositivos de uso clínico: implantes, materiales inertes, metales y cerámicos.

Ingeniería Genética: decodificación del Código Genético, uso de organismos transgénicos y clonación.

Biomedicina: prótesis con tecnología 3D. Cyborgs: sistema Hombre- Máquina: Marcapasos, Implante Coclear, Ojo Biónico.

En el campo de la exploración espacial las fronteras se siguen expandiendo a misiones estelares a otros planetas. Tenemos la sonda espacial Juno dedicada al estudio del planeta Júpiter, lanzada el 5 de agosto de 2011, la cual llegó a la órbita del mismo el 5 de julio de 2016.

Y actualmente dos Ingenieros argentinos participan de la exploración de Marte: ellos son el Ing. Miguel San Martín que desarrolló el sistema informático para el vehículo rover Curiósity, de la Misión "Mars 2011” la cual viajó para tomar muestras de suelo y rocas, para determinar condiciones de vida en el planeta rojo.

Y el Ing. Daniel Romero, encargado del instrumental del vehículo rover Perseverance de la Misión "Mars 2020" que viajó 29/07/20; y que cuenta con otros instrumentos: Moxie para fabricar oxígeno, Sherloc para encontrar materia orgánica en la superficie, Pixl para estudiar la química del suelo marciano y Esye un dron para observar los lugares a recorrer.

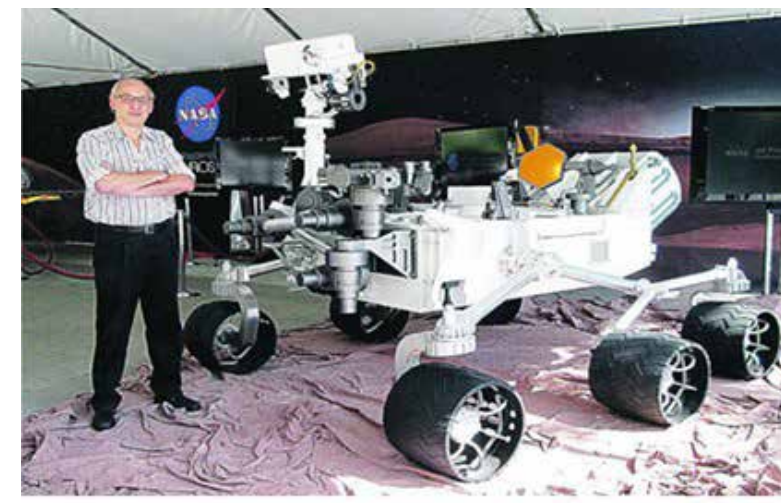

Ing. Miguel San Martín y el rover Curiosity. Misión Mars 2011

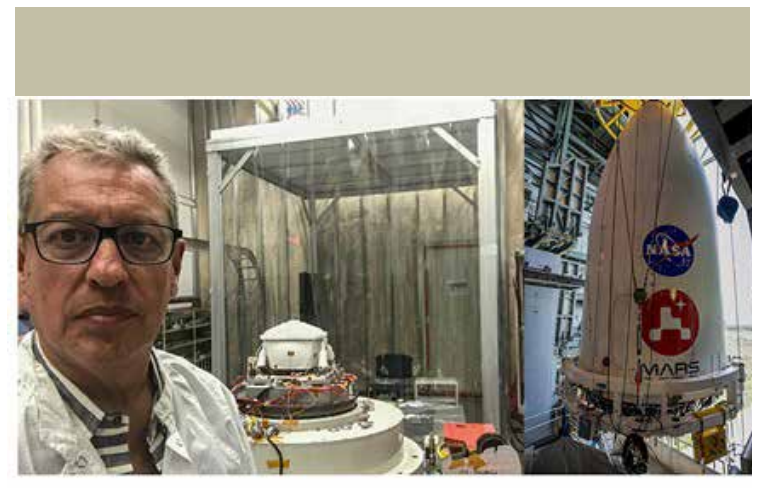

Ing. Daniel Romero y el rover Perseverance. Misión Mars 2020 


\section{Un mundo y dos Globalizaciones: revolución tecno-industrial 4.o}

Esta segunda globalización tecnológica resulta del proceso de incorporación, expansión e integración de todos los componentes de la vida local de los países a una gran red mundial tecnológica. Para ello combina infraestructura física con software, sensores, nanotecnología, tecnología digital de comunicaciones, todo lo cual modificó el mundo del empleo incidiendo en las industrias de todo el planeta.

Estamos en una época de mayor interconexión mundial en la historia de la humanidad, donde la Ingeniería se ha puesto al frente de las transformaciones tecnológicas que han tenido impacto a nivel mundial y regional, y dado que las barreras aduaneras cada vez son menores, esto posibilitó el turismo de masas, con flujos de personas, mercancías e información, con miles de vuelos que conectan las partes más dispares del mundo. Todo lo cual determinó la rápida transmisión del coronavirus SARS-CoV-2 que se propagó por todos los rincones del planeta; podemos considerar que la pandemia del COVID-19 es hija de la globalización, ya que la conexión digital la convierte en una experiencia compartida en tiempo real.

La globalización en este período de pandemia nos demostró que un virus pudo cambiarnos la vida a todos, (lado oscuro) pero también nos enseñó que la comunicación y la cooperación entre los diferentes países generaron nuevos desarrollos que pueden beneficiar a todos.

\section{El mundo del Covid 19: lo virtual y lo tecnológico en la nueva normalidad}

Esta nueva situación socio sanitaria impuesta por la pandemia (OMS) obligó a nuevas costumbres, que apoyadas en la investigación y el conocimiento científico generado a cada momento, dio impulso al desarrollo de nuevas tecnologías, y al mismo tiempo con el uso masivo de la virtualidad, permitió sostener las actividades educativas, comerciales, artísticas y asistenciales en toda la gama de acciones que hacen al quehacer humano; esto dio en llamarse la nueva normalidad.

La Ciberescuela: posibilita que los estudiantes en los diferentes grados de escolaridad, puedan recibir sus clases presenciales con un aula virtual donde se comparten documentos, videos o tiene lugar una conversación en una plataforma web; 
también se realizan actividades académicas de perfeccionamiento con la ayuda de tutores; de esta manera se enseña y se aprende desde el hogar. Para ello se emplean diferentes plataformas educativas: Cerebriti, Office 365, Educ.Ar. Las relaciones personales y laborales se establecen por: videollamadas: Skype, Messenger, Jitsi y por videoconferencias: Zoom, Meet.

En este tiempo particular cobra auge el Teletrabajo: es decir toda actividad laboral que puede realizarse por medio del uso de las tecnologías. Ejemplo de ella es la Telemedicina donde a través de Internet y por el uso de un chat se realizan consultas a la distancia entre un Médico asignado y el Paciente, quien puede hacerle las consultas que estime oportunas. Entre sus beneficios esta la comodidad de ver y escuchar al médico desde el hogar a través del ordenador o del móvil, con el consiguiente ahorro de tiempo de desplazamiento y de espera.

Robótica: en algunos hospitales los robots son usados para desinfectar habitaciones, llevar medicinas y asistir a pacientes, trabajadores y médicos en los diferentes sectores de los hospitales y en los quirófanos. Los Robots son autónomos y van aprendiendo en cada recorrido, volviéndose cada vez más eficientes y veloces: tienen como ventaja que no sufren agotamiento o estrés, aun trabajando las 24 horas del día. Ejemplo de ello es Tommy, el robot enfermero, que atiende a pacientes contagiados del Covid-19 en el Hospital de Varese, región de Lombardía. Asimismo, el uso de drones inteligentes para desinfección, transporte y entrega de medicinas.

Softwares: con tecnología de reconocimiento facial y para detección de temperatura; de esta manera se busca identificar a las personas que presentan fiebre, ya que tienen más probabilidades de tener el virus. También están utilizando un software para escanear multitudes en busca de las personas que no usan máscaras o barbijos para protegerse y evitar la diseminación del virus.

Aplicaciones: en este contexto, hay varias gratuitas que permiten conectarse virtualmente, con el objetivo de lograr que las personas se relacionen por medio de la red. Whats App es uno de los servicios de mensajería más populares con más de 2 mil millones de usuarios en el mundo, permitiendo hacer videollamadas para un encuentro virtual en estos momentos de aislamiento social. Surgió así un sinnúmero 
de aplicaciones con diversos fines: Recreativo: TikTok, Instagram, FaceApp; asociado al mundo de las finanzas: Uala, Brubank, Rebanking; propuestas de turismo sin salir de casa: tours virtuales por museos; la entretención vía streaming, y los espectáculos: Netflix, Disney, Amazon Prime Video; que junto con la descarga gratuita de libros o de películas nos invitan a permanecer en casa.

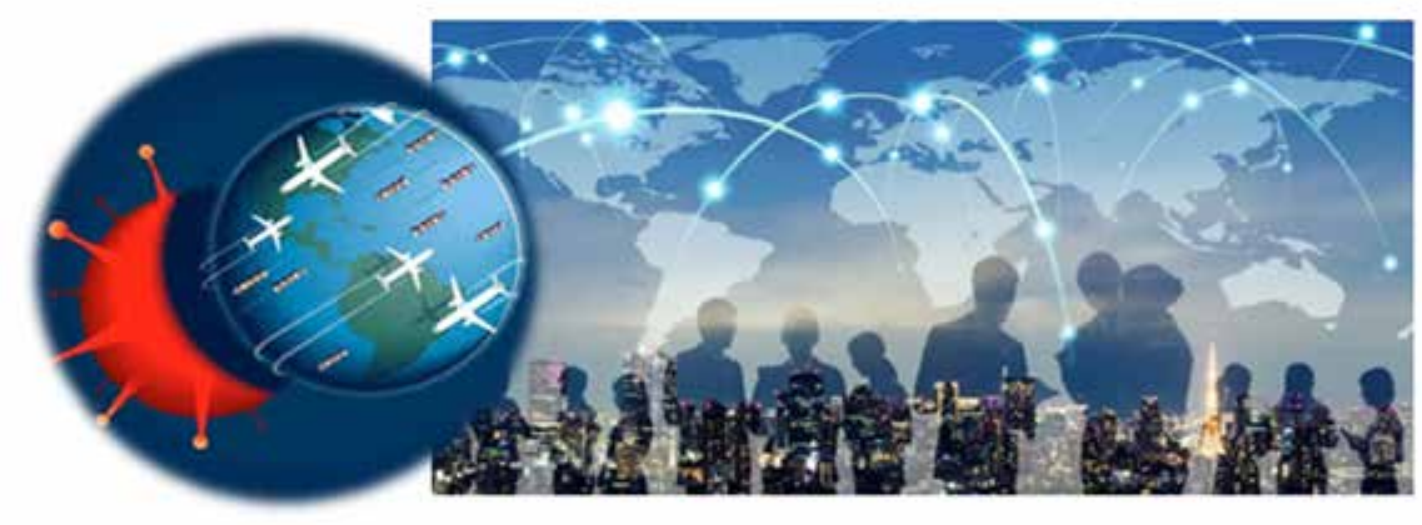

\section{Conclusiones:}

La Ingeniería, y las relaciones que establece con la Ciencia y la Tecnología, fueron el punto de partida del presente trabajo; y desde allí hemos visto como la transformación de la realidad tuvo lugar desde tiempos remotos hasta el actual tercer milenio.

Esto fue posible a impulsos de la Ciencia y la Tecnología, que capitalizados por la Ingeniería, lograron un camino de innovación y desarrollo en pos del progreso de la humanidad La revolución del conocimiento en diferentes campos de la ciencia, estrechamente unidos a su aplicación tecnológica, potenció el desarrollo de la Ingeniería, que de esta forma contribuyó a crear una nueva realidad con una gran impronta en las comunicaciones, lo que desembocó en el fenómeno de la globalización y una neocultura.

La particular situación de pandemia que atravesamos, ha generado un nuevo salto en nuestro viaje, donde planteado éste desafío a la imaginación y capacidad de acción; la Ciencia y la Tecnología han debido redoblar esfuerzos junto con la Ingeniería; para dar respuesta a los nuevos retos y de esta manera lograr dominar la naturaleza del virus, para mitigar su accionar y así preservar la vida en el planeta azul. 


\section{Bibliografía:}

Andalia R.C; “Ciencia y Tecnología en la Sociedad. Perspectiva histórico-conceptual” ACIMED Vol. 9 No 1. 2001. La Habana. Cuba.

Baca Urbina, G. et al. "Introducción a la Ingeniería Industrial” 20 Ed. Patria. 2014. México.

Corvalán R.E; Estebanéz M. E; Cuevas A.S.”Vinculación científica Tecnológica en una Universidad Argentina” Editorial Académica Española. 2014. Deutschland. Alemania.

Dettmer J; “Ciencia, Tecnología e Ingeniería” Revista de Educación Superior. Vol. XXXII. No 128. 2003. UNAM. México.

Perusset M; “Tecnología e Ingeniería en el Siglo XXI: una conexión cada vez más estrecha” Revista Proyecciones Vol. $11 \mathrm{~N}^{0}$ 2. 2013. UTN. Bs. As. Argentina.

Sobrevila, M. A. “Ingeniería General”. Editorial Alsina. 2011. Bs. As. Argentina.

Valencia Giraldo A; "La relación entre la Ingeniería y la Ciencia” Revista Facultad de Ingeniería. $\mathrm{N}^{\mathrm{o}}$ 31. 2004. Antioquia. Colombia. 